\title{
Hilbert domains that admit a quasi-isometric embedding into Euclidean space
}

\author{
Bruno Colbois and Patrick Verovic \\ (Communicated by M. Henk)
}

\begin{abstract}
We prove that a Hilbert domain which admits a quasi-isometric embedding into a finite-dimensional normed vector space is actually a convex polytope.
\end{abstract}

Key words. Primary: global Finsler geometry, Secondary: convexity.

\section{Introduction}

A Hilbert domain in $\mathbb{R}^{m}$ is a metric space $\left(\mathcal{C}, d_{\mathcal{C}}\right)$, where $\mathcal{C}$ is an open bounded convex set in $\mathbb{R}^{m}$ and $d_{\mathcal{C}}$ is the distance function on $\mathcal{C}$ - called the Hilbert metric - defined as follows. Given two distinct points $p$ and $q$ in $\mathcal{C}$, let $a$ and $b$ be the intersection points of the straight line defined by $p$ and $q$ with $\partial \mathcal{C}$ so that $p=(1-s) a+s b$ and $q=(1-t) a+t b$ with $0<s<t<1$. Then

$$
\begin{aligned}
& d_{\mathcal{C}}(p, q):=\frac{1}{2} \ln [a, p, q, b], \quad \text { where } \\
& {[a, p, q, b]:=\frac{1-s}{s} \times \frac{t}{1-t}>1}
\end{aligned}
$$

is the cross ratio of the 4-tuple of ordered collinear points $(a, p, q, b)$. We complete the definition by setting $d_{\mathcal{C}}(p, p):=0$.

The metric space $\left(\mathcal{C}, d_{\mathcal{C}}\right)$ thus obtained is a complete non-compact geodesic metric space whose topology is the one induced by the canonical topology of $\mathbb{R}^{m}$ and in which the affine open segments joining two points of the boundary $\partial \mathcal{C}$ are geodesic lines. For further information about Hilbert geometry, we refer to $[4,5,9,11]$ and the excellent introduction [15] by Socié-Méthou.

The two fundamental examples of Hilbert domains $\left(\mathcal{C}, d_{\mathcal{C}}\right)$ in $\mathbb{R}^{m}$ correspond to the case when $\mathcal{C}$ is an ellipsoid, which gives the Klein model of $m$-dimensional hyperbolic 


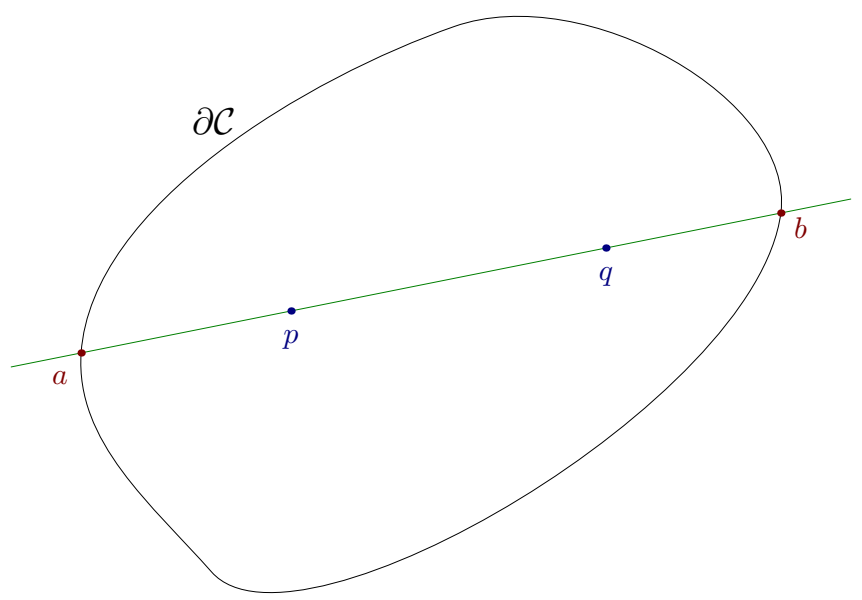

geometry (see for example [15, first chapter]), and the case when the closure $\overline{\mathcal{C}}$ is a $m$ simplex for which there exists a norm $\|\cdot\|_{\mathcal{C}}$ on $\mathbb{R}^{m}$ such that $\left(\mathcal{C}, d_{\mathcal{C}}\right)$ is isometric to the normed vector space $\left(\mathbb{R}^{m},\|\cdot\|_{\mathcal{C}}\right)$ (see [8, pages 110-113] or [14, pages 22-23]).

Much has been done to study the similarities between Hilbert and hyperbolic geometries (see for example [7], [16] or [1]), but little literature deals with the question of knowing to what extend a Hilbert geometry is close to that of a normed vector space in terms of quasi-isometric embeddings which are defined as follows (see Definition 8.14 in [3], page 138):

Definition. Given real numbers $A \geq 1$ and $B \geq 0$, a metric space $(S, d)$, and a normed vector space $(V,\|\cdot\|)$, a map $f: S \rightarrow V$ is said to be a $(A, B)$-quasi-isometric embedding if

$$
\frac{1}{A} d(p, q)-B \leq\|f(p)-f(q)\| \leq A d(p, q)+B
$$

for all $p, q \in S$.

Definition. With this definition, a map $f: S \rightarrow V$ is called a Lipschitz equivalence if it is a bijection that is a $(A, 0)$-quasi-isometric embedding for some $A \geq 1$.

Let us then mention three results which are relevant for our present work.

Theorem 1.1 ([10], Theorem 2). A Hilbert domain $\left(\mathcal{C}, d_{\mathcal{C}}\right)$ in $\mathbb{R}^{m}$ is isometric to some normed vector space if and only if $\mathcal{C}$ is the interior of a m-simplex.

Theorem 1.2 ([6], Theorem 3.1). If $\mathcal{C}$ is an open convex polygonal set in $\mathbb{R}^{2}$, then $\left(\mathcal{C}, d_{\mathcal{C}}\right)$ is Lipschitz equivalent to the Euclidean plane.

Theorem 1.3 ([2], Theorem 1.1. See also [17]). If $\mathcal{C}$ is an open set in $\mathbb{R}^{m}$ whose closure $\overline{\mathcal{C}}$ is a convex polytope, then $\left(\mathcal{C}, d_{\mathcal{C}}\right)$ is Lipschitz equivalent to Euclidean $m$-space. 
Recall that a convex polytope in $\mathbb{R}^{m}$ (called a convex polygon when $m:=2$ ) is the convex hull of a finite set of points whose affine span is the whole space $\mathbb{R}^{m}$. In the light of these facts, it is natural to ask whether the converse of Theorem 1.3 - which generalizes Theorem 1.2 in higher dimensions — holds. In other words, if a Hilbert domain $\left(\mathcal{C}, d_{\mathcal{C}}\right)$ in $\mathbb{R}^{m}$ admits a quasi-isometric embedding into a normed vector space, what can be said about $\mathcal{C}$ ? The answer to that question is given by the following result which asserts that the converse of Theorem 1.3 is actually true:

Theorem 1.4. If a Hilbert domain $\left(\mathcal{C}, d_{\mathcal{C}}\right)$ in $\mathbb{R}^{m}$ admits a quasi-isometric embedding into a finite-dimensional normed vector space $(V,\|\cdot\|)$, then $\mathcal{C}$ is the interior of a convex polytope.

\section{Proof of Theorem 1.4}

The proof of Theorem 1.4 is based on an idea developed by Foertsch and Karlsson in their paper [10]. For this purpose, let us first introduce a definition:

Definition. Given a convex set $\mathcal{C}$ in $\mathbb{R}^{m}$ and $x, y \in \partial \mathcal{C}$, we will say that the points $x$ and $y$ are neighbors if the line segment $[x, y]$ between them is contained in the boundary $\partial \mathcal{C}$.

To establish Theorem 1.4, we need the following fact due to Karlsson and Noskov:

Theorem 2.1 ([12], Theorem 5.2). Let $\left(\mathcal{C}, d_{\mathcal{C}}\right)$ be a Hilbert domain in $\mathbb{R}^{m}$ and $x, y \in \partial \mathcal{C}$ such that $x$ and $y$ are not neighbors. Then, fixing any point $p_{0} \in \mathcal{C}$, there exists a constant $K\left(p_{0}, x, y\right)>0$ such that for any sequences $\left(x_{n}\right)_{n \in \mathbf{N}}$ and $\left(y_{n}\right)_{n \in \mathbf{N}}$ in $\mathcal{C}$ that converge respectively to $x$ and $y$ in $\mathbb{R}^{m}$ one has

$$
d_{\mathcal{C}}\left(x_{n}, y_{n}\right) \geq d_{\mathcal{C}}\left(x_{n}, p_{0}\right)+d_{\mathcal{C}}\left(y_{n}, p_{0}\right)-K\left(p_{0}, x, y\right)
$$

for sufficiently large $n$.

Now, here is the key result which gives the proof of Theorem 1.4:

Proposition 2.1. Let $\left(\mathcal{C}, d_{\mathcal{C}}\right)$ be a Hilbert domain in $\mathbb{R}^{m}$ which admits a $(A, B)$-quasiisometric embedding into a finite-dimensional normed vector space $(V,\|\cdot\|)$ for some real constants $A \geq 1$ and $B \geq 0$. Then, if $N=N(A,\|\cdot\|)$ denotes the maximum number of points in the ball $\{v \in V \mid\|v\| \leq 2 A\}$ whose pairwise distances with respect to $\|\cdot\|$ are greater than or equal to $1 /(2 A)$, and if $X \subseteq \partial \mathcal{C}$ consists of pairwise non-neighboring points, we have $\operatorname{card}(X) \leq N$.

Definition. The finiteness of the dimension of $V$ is essential here since it yields the compactness of the ball $\{v \in V \mid\|v\| \leq 2 A\}$, insuring that $N$ is well defined (indeed, if there were an infinite number of points in this ball whose pairwise distances with respect to $\|\cdot\|$ are greater than or equal to $1 /(2 A)$, then these points would have an accumulation point 
in this ball, which is impossible). On the other hand, it is worth remembering that any metric space $(S, d)$ can be isometrically embedded into the infinite-dimensional space of bounded real functions on $S$ endowed with the uniform norm.

Proof of Proposition 2.1. Let $f: \mathcal{C} \rightarrow V$ such that

$$
\frac{1}{A} d_{\mathcal{C}}(p, q)-B \leq\|f(p)-f(q)\| \leq A d_{\mathcal{C}}(p, q)+B
$$

for all $p, q \in \mathcal{C}$. First of all, up to translations, we may assume that $0 \in \mathcal{C}$ and $f(0)=0$.

Then suppose that there exists a subset $X$ of the boundary $\partial \mathcal{C}$ such that $[x, y] \nsubseteq \nexists \mathcal{C}$ for all $x, y \in X$ with $x \neq y$ and $\operatorname{card}(X) \geq N+1$. So, pick $N+1$ distinct points $x_{1}, \ldots, x_{N+1}$ in $X$, and for each $k \in\{1, \ldots, N+1\}$, let $\gamma_{k}:[0,+\infty) \rightarrow \mathcal{C}$ be a geodesic of $\left(\mathcal{C}, d_{\mathcal{C}}\right)$ that satisfies $\gamma_{k}(0)=0, \lim _{t \rightarrow+\infty} \gamma_{k}(t)=x_{k}$ in $\mathbb{R}^{m}$ and $d_{\mathcal{C}}\left(0, \gamma_{k}(t)\right)=t$ for all $t \geq 0$.

This implies that for all integer $n \geq 1$ and every $k \in\{1, \ldots, N+1\}$, we have

$$
\left\|\frac{f\left(\gamma_{k}(n)\right)}{n}\right\| \leq A+\frac{B}{n}
$$

from the second inequality in Equation 2.1 with $p:=\gamma_{k}(n)$ and $q:=0$. On the other hand, Theorem 2.1 yields the existence of some integer $n_{0} \geq 1$ such that

$$
d_{\mathcal{C}}\left(\gamma_{i}(n), \gamma_{j}(n)\right) \geq 2 n-K\left(0, x_{i}, x_{j}\right)
$$

for all integer $n \geq n_{0}$ and every $i, j \in\{1, \ldots, N+1\}$ with $i \neq j$, and hence

$$
\left\|\frac{f\left(\gamma_{i}(n)\right)}{n}-\frac{f\left(\gamma_{j}(n)\right)}{n}\right\| \geq \frac{2}{A}-\frac{1}{n}\left(\frac{K\left(0, x_{i}, x_{j}\right)}{A}+B\right)
$$

from the first inequality in Equation 2.1 with $p:=\gamma_{i}(n)$ and $q:=\gamma_{j}(n)$.

Now, fixing an integer $n \geq n_{0}+A B+\max \left\{K\left(0, x_{i}, x_{j}\right) \mid i, j \in\{1, \ldots, N+1\}\right\}$, we get

$$
\left\|\frac{f\left(\gamma_{k}(n)\right)}{n}\right\| \leq 2 A
$$

for all $k \in\{1, \ldots, N+1\}$ by Equation 2.2 together with

$$
\left\|\frac{f\left(\gamma_{i}(n)\right)}{n}-\frac{f\left(\gamma_{j}(n)\right)}{n}\right\| \geq \frac{1}{2 A}
$$

for all $i, j \in\{1, \ldots, N+1\}$ with $i \neq j$ by Equation 2.3. But this contradicts the definition of $N=N(A,\|\cdot\|)$.

Owing to Proposition 2.1, Theorem 1.4 is then a straightforward consequence of the following:

Proposition 2.2. Let $\mathcal{C}$ be an open bounded convex set in $\mathbb{R}^{m}$. Then $\overline{\mathcal{C}}$ is a convex polytope if and only if there exists an integer $N \geq 1$ such that every set $X \subseteq \partial \mathcal{C}$ made up of pairwise non-neighboring points satisfies $\operatorname{card}(X) \leq N$. 
The proof of Proposition 2.2 will use two useful results.

Lemma 2.1. In a convex set $\mathcal{C}$ in $\mathbb{R}^{2}$, any extreme point (hence in $\partial \mathcal{C}$ ) has at most two other extreme points as neighbors.

Theorem 2.2 ([13], Theorem 4.7). Let $P$ be a convex set in $\mathbb{R}^{m}$ and $p \in \stackrel{\circ}{P}$. Then $P$ is a convex polyhedron if and only if all its plane sections containing $p$ are convex polyhedra.

Here, as usual, a convex polyhedron in $\mathbb{R}^{m}$ is the intersection of a finite number of closed half-spaces.

Proof of Lemma 2.1. Suppose there exists an extreme point $p_{0}$ of $\mathcal{C}$ that has three other extreme points $m, p$ and $q$ in $\mathcal{C}$ as neighbors. Up to translations, we may assume that $p_{0}=$ 0 . Since $[0, p]$ and $[0, q]$ are contained in the boundary $\partial \mathcal{C}$, the straight lines $(0 p)$ and $(0 q)$ are supporting lines to $\mathcal{C}$, and hence $\mathcal{C}$ lies in the half-cone $\{\lambda p+\mu q \mid \lambda \geq 0$ and $\mu \geq 0\}$.

In particular, we can write $m=\lambda_{0} p+\mu_{0} q$ for some $\lambda_{0}>0$ and $\mu_{0}>0$ (being extreme in $\mathcal{C}$, the points $m$ and $p$ can neither satisfy $m \in[0, p]$, nor $p \in[0, m]$; therefore $\lambda_{0}$ cannot vanish, and the same holds for $\mu_{0}$ ).

Now the open convex hull $\{\lambda p+\mu q \mid \lambda>0, \mu>0$ and $\lambda+\mu=1\}$ of the points $0, p, q \in \mathcal{C}$ is contained in $\stackrel{\circ}{\mathcal{C}}$, which yields $m /\left(\lambda_{0}+\mu_{0}\right) \in \stackrel{\circ}{\mathcal{C}}$. Therefore, since $m /\left(\lambda_{0}+\right.$ $\left.\mu_{0}\right) \in[0, m]$, we get $[0, m] \nsubseteq \partial \mathcal{C}$, contradicting the fact that 0 and $m$ are neighbors.

Proof of Proposition 2.2. If $\mathcal{C}$ is a convex polytope, then any two points in $\partial \mathcal{C}$ that are not neighbors belong to two different 2-dimensional faces of $\mathcal{C}$. Thus, every set $X \subseteq \partial \mathcal{C}$ consisting of pairwise non-neighboring points satisfies $\operatorname{card}(X) \leq N$, where $N$ denotes the number of 2-dimensional faces of $\mathcal{C}$. On the other hand, if $\mathcal{C}$ is not a convex polytope, then Theorem 2.2 implies that there exists a plane section of $\mathcal{C}$ which is not a convex polygon. So, we may assume that $m=2$.

Since any compact convex set in a finite-dimensional real vector space is the convex hull of its extreme points (Minkowski's Theorem), $\mathcal{C}$ has infinitely many extreme points. Then, using Lemma 2.1, one can construct by induction a sequence $\left(x_{n}\right)_{n \in \mathbf{N}}$ of extreme points in $\mathcal{C}$ such that for all $n \in \mathbf{N}$ the point $x_{n+1}$ has no neighbors in $\left\{x_{0}, \ldots, x_{n}\right\}$, and hence the set $X:=\left\{x_{n} \mid n \in \mathbf{N}\right\} \subseteq \partial \mathcal{C}$ is infinite and is made up of pairwise non-neighboring points. This proves Proposition 2.2.

Acknowledgment. The authors are very grateful to the referee for his relevant suggestions.

\section{References}

[1] Y. Benoist, A survey on divisible convex sets. In: Geometry, analysis and topology of discrete groups, volume 6 of Adv. Lect. Math. (ALM), 1-18, Int. Press, Somerville, MA 2008. MR2464391 (2010h:52013) Zbl 1154.22016 
[2] A. Bernig, Hilbert geometry of polytopes. Arch. Math. (Basel) 92 (2009), 314-324. MR2501287 (2010c:53106) Zbl 1171.53046

[3] M. R. Bridson, A. Haefliger, Metric spaces of non-positive curvature. Springer 1999. MR1744486 (2000k:53038) Zbl 0988.53001

[4] H. Busemann, The geometry of geodesics. Academic Press 1955. MR0075623 (17,779a) Zbl 0112.37002

[5] H. Busemann, P. J. Kelly, Projective geometry and projective metrics. Academic Press 1953. MR0054980 (14,1008e) Zbl 0052.37305

[6] B. Colbois, C. Vernicos, P. Verovic, Hilbert geometry for convex polygonal domains. Technical report, University of Neuchâtel, 2008.

[7] B. Colbois, P. Verovic, Hilbert geometry for strictly convex domains. Geom. Dedicata 105 (2004), 29-42. MR2057242 (2005e:53111) Zbl 1078.52002

[8] P. de la Harpe, On Hilbert's metric for simplices. In: Geometric group theory, Vol. 1 (Sussex, 1991), volume 181 of London Math. Soc. Lecture Note Ser., 97-119, Cambridge Univ. Press 1993. MR1238518 (94i:52006) Zbl 0832.52002

[9] D. Egloff, Uniform Finsler Hadamard manifolds. Ann. Inst. H. Poincaré Phys. Théor. 66 (1997), 323-357. MR1456516 (98c:53079) Zbl 0919.53020

[10] T. Foertsch, A. Karlsson, Hilbert metrics and Minkowski norms. J. Geom. 83 (2005), 22-31. MR2193224 (2007e:51021) Zbl 1084.52008

[11] W. Goldman, Projective geometry on manifolds. Lecture notes, University of Maryland, 1988.

[12] A. Karlsson, G. A. Noskov, The Hilbert metric and Gromov hyperbolicity. Enseign. Math. (2) 48 (2002), 73-89. MR1923418 (2003f:5361) Zbl 1046.53026

[13] V. Klee, Some characterizations of convex polyhedra. Acta Math. 102 (1959), 79-107. MR0105651 (21 \#4390) Zbl 0094.16802

[14] R. D. Nussbaum, Hilbert's projective metric and iterated nonlinear maps. Mem. Amer. Math. Soc. 75 (1988), iv+137. MR961211 (89m:47046) Zbl 0666.47028

[15] É. Socié-Méthou, Comportements asymptotiques et rigidités des géométries de Hilbert. $\mathrm{PhD}$ thesis, University of Strasbourg, 2000.

[16] C. Vernicos, Introduction aux géométries de Hilbert. In: Actes de Séminaire de Théorie Spectrale et Géométrie. Vol. 23. Année 2004-2005, 145-168. MR2270228 (2007m:51011) Zbl 1100.53031

[17] C. Vernicos, Lipschitz characterization of polytopal Hilbert geometries. Technical report, University of Maynooth, 2008.

Received 12 May, 2009; revised 12 July, 2009

B. Colbois, Université de Neuchâtel, Institut de mathématique, Rue Émile Argand 11, Case postale 158, 2009 Neuchâtel, Switzerland

Email: bruno.colbois@unine.ch

P. Verovic, UMR 5127 du CNRS \& Université de Savoie, Laboratoire de mathématique, Campus scientifique, 73376 Le Bourget-du-Lac Cedex, France

Email: verovic@univ-savoie.fr 